\title{
INFLUENCE OF VibRATION TRAINING ON ENERGY Expenditure In ACTIVE Men
}

\author{
Marzo E. Da Silva, Juan M. Fernandez, Eduardo Castillo, Victor M. Nuñez, \\ Diana M. Vaamonde, Maria S. Poblador, and Jose L. Lancho
}

Morphological Sciences Department, School of Medicine, University of Córdoba, Córdoba, Spain.

\begin{abstract}
Da Silva, M.E., J.M. Fernandez, E. Castillo, V.M. Nuñez, D.M. Vaamonde, M.S. Poblador, and J.L. Lancho. Influence of vibration training on energy expenditure in active men. J. Strength Cond. Res. 21(2):470-475. 2007.-The aim of the present study was to analyze the effect of whole-body vibration on energy expenditure, as well as on exercise intensity, during and immediately after a typical set of exercises for muscle hypertrophy in physically active subjects. Seventeen male university students (mean age $18.3 \pm 0.24$ years) volunteered to perform 2 different training exercises: half squat (HS), and half squat with vibration (HSV). Both exercises were performed by all subjects on the vibration platform (with vibration only for HSV), the sequence order being assigned randomly. Energy expenditure (EE), respiratory exchange ratio, perceived exertion $(\mathrm{PE})$, and heart rate were recorded for baseline, exercise, and short-recovery conditions. Training consisted of 5 sets of 10 repetitions of HS and HSV, with a 2-minute recovery interval between sets. Analysis of variance with repeated measurements and Bonferroni correction, as well as effect size were used for statistical calculations. Results indicated that $\mathrm{EE}$ and $\mathrm{PE}$ were significantly higher in the HSV group, during both exercise and recovery. Heart rate did not differ significantly between groups. Thus, it would appear that HS strength training could be rendered more energy-efficient through the addition of vibration. Moreover, it would be feasible to introduce vibration exercises into regular training programs, particularly those whose key objective is muscle hypertrophy along with fat reduction.
\end{abstract}

KEY WORDS. perceived exertion, muscular strength, oxygen consumption, respiratory exchange ratio

\section{INTRODUCTION}

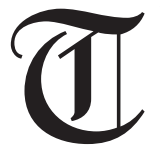

he loss of a steady state resulting in a positive energy balance includes metabolic adjustments that might force biological changes, such as adipogenesis and an increased risk of nontransmittable chronic diseases (39). The long-term efficiency of most dietary strategies aimed at achieving a negative energy balance remains doubtful if sedentary lifestyles are not modified (40). The use of low-calorie diets, either alone or in conjunction with aerobic exercise, usually promotes the loss of adipose tissue but may also induce some loss of fat-free mass, thus reducing the resting metabolic rate (RMR) (10). Resistance training, in contrast, increases fat-free mass and enhances catecholamine secretion, thus maintaining or even raising the RMR $(10,11,41)$.

Whole-body vibration (WBV) training is increasingly being used to improve performance $(4-6,13,16,23)$ and to treat neuromuscular (31) and bone pathologies $(34,38)$. It has been reported that 5 weeks of WBV training significantly improve strength and power in comparison to the same training carried out without vibration; at the same time, WBV training induces trophic improvements in muscle (33). Moreover, Bosco et al. (6) report that WBV training prompts an increase in plasma concentrations of testosterone and growth hormone, and a decrease in cor- tisol levels, thus affording metabolic benefits in addition to increased motor unit efficiency.

In terms of bioenergetics, it has been demonstrated that resistance training involving WBV produces an increase in oxygen uptake, and therefore in energy expenditure (EE) in comparison to the same exercise without WBV (32). It has further been reported that this increase can be parametrically controlled by frequency, amplitude, and the external load applied during WBV (32). Relatively few studies, however, address the effect of additional WBV on energy expenditure during a set of half squat (HS) exercises, which are common in programs designed to increase strength and muscle hypertrophy. To the best of our knowledge, no direct data are available on the possible energy benefits of this type of training.

Since WBV has been shown to increase energy expenditure, it was hypothesized that the application of vibration to HS exercises typically used to produce muscle hypertrophy would provide enhanced metabolic power. The purpose of the present study, therefore, was to analyze the influence of whole-body vibration on energy expenditure, during both the active exercise phase and the short-recovery phase.

\section{MeTHODS}

\section{Experimental Approach to the Problem}

Participants underwent an initial 2-week period of familiarization with HS training, after which all subjects performed 2 different training sessions in a counterbalanced crossover design, HS and HSV, separated by intervals of 48 to 72 hours. The order in which each participant underwent the 2 different sessions was assigned randomly. Each participant acted as his own control. At each session, energy expenditure (EE) was measured (kcal per minute and kcal per session), together with the respiratory exchange ratio ( $R E R$ ), in order to determine the effect of WBV on these variables. Heart rate (HR) and perceived exertion (PE) were also recorded, since these variables are representative of effort intensity.

\section{Subjects}

Seventeen healthy male university students participated voluntarily in this research project. All of them were physically active. A physician reviewed their medical records, and volunteers who had previous severe neuromuscular or bone pathologies, epilepsy, or a history of chronic or metabolic disease were excluded. None of the subjects had any previous experience with WBV training; however, all subjects had been performing resistance training for at least 6 months prior to the study, with a minimum frequency of 2 sessions per week and a maximum of 4 . Over the whole experimental period, subjects refrained from performing any exercise other than that required for 
TABLE 1. Physical and body composition characteristics of the subjects.

\begin{tabular}{lrc}
\hline \multicolumn{1}{c}{ Variable } & Mean & $S D$ \\
\hline Age (y) & 18.3 & 0.24 \\
Height $(\mathrm{cm})$ & 179.0 & 7.2 \\
Mass (kg) & 76.7 & 9.0 \\
Body fat (\%) & 16.4 & 4.2 \\
Body fat (kg) & 12.8 & 4.1 \\
Fat-free mass (\%) & 83.6 & 4.2 \\
Fat-free mass (kg) & 63.9 & 6.0 \\
Resting metabolic rate (kcal per 24 h) & 1678.0 & 317.4 \\
Heart rate (b·min ${ }^{-1}$ ) & 72.4 & 14.2 \\
Systolic blood pressure & 129.4 & 8.8 \\
Diastolic blood pressure & 79.7 & 6.7 \\
\hline
\end{tabular}

this research. Prior to participation, and in accordance with procedures approved by the University Institutional Review Board for human subjects, each subject was informed-both verbally and in writing-of all procedures, potential risks, and benefits associated with the study, and signed an informed consent form prior to inclusion. After an overnight fast of not less than 10 hours, subjects arrived at the laboratory, where they rested for 20 minutes. At the end of this period, their resting metabolic rate was measured using an automatic breath-by-breath gas analysis system (CPX, MedGraphics, St. Paul, MN). Blood pressure and resting heart rate were also measured. Body composition was assessed by skinfold thickness, while body density $\left(\mathrm{g} \cdot \mathrm{cm}^{-3}\right)$ was calculated using the Durnin and Womersley equations (17); percentage of body fat was determined using the equations formulated by Brozek et al. (9). Physical and body-composition characteristics of the subjects are shown in Table 1.

\section{Ten Repetition Maximum Testing}

To calculate the 10 repetition maximum (10RM) for each subject, a standardized procedure was used for the submaximum strength test proposed by Kraemer and Fry (24). The mass of all weight plates that were used was determined with a precision scale. The actual mass of all plates was used to calculate 10RM in the HS exercise. Several strategies were implemented in order to minimize possible errors in 10RM testing: (a) all subjects were given identical information about how to perform the tests and how the data would be assessed, (b) individual exercise techniques of subjects were monitored and corrected as required throughout the training sessions, and (c) all subjects received verbal encouragement during testing. For a repetition to be successful, the range of motion typical for this exercise had to be completed. For this purpose, subjects were placed in a half-squat position, with shoulders touching the bar; the starting knee angle for movement execution was set at $90^{\circ}$. During each repetition, the subjects started from the $90^{\circ}$ flexed-knee position and performed concentric extension of the leg muscles (hip, knee, and ankle) until reaching full extension at $180^{\circ}$, thereafter returning to the initial $90^{\circ}$ position, with no pauses between the 10 repetitions.

\section{Familiarization Training}

During the 2 weeks of familiarization training, subjects performed HS exercises on a multipower machine (GervaSport, Madrid, Spain) at a rate of 1 repetition every 4 seconds (2-second eccentric phase plus 2 -second concentric phase), controlled by a digital metronome. The training protocol is described in Table 2. Prior to each training session, subjects performed 5 minutes of standing stretch exercises. To be able to perform all 10-repetition sets required, subjects were allowed to decrease slightly (by a maximum of $10 \%$ ) the load calculated using the 10RM test. To guarantee that all sets were performed with maximal intensity during this period, an adaptation of the categorization of effort (CE) system was used (19). The CE score is expressed by the relationship between the number of repetitions carried out per set (those which the subject manages to do) and those required by the training; this relationship thus indicates exercise intensity. Scores are divided into 3 categories: nonmaximum $\mathrm{CE}$ (the subject is capable of doing more repetitions than those imposed), maximum CE (the subject cannot do any more repetitions than those imposed) and supramaximum CE (the subject does not manage to complete the repetitions required by the training). The $\mathrm{CE}$ system was adapted to the bout of exercises used here, i.e., if $\mathrm{CE}$ were maximum the subject would not be able to perform a single further repetition after completing the last set of exercises (Table 2).

\section{Treatment Procedure}

A protocol of 10 repetitions maximum (10RM) was used for HS exercises to stimulate a relatively high metabolic level. For assessment purposes, all subjects were randomly tested while performing HS and HSV exercises (Table 2 ). Both exercises were performed on a vibration platform (NEMES, Ergotest, Italy); however, vertical sinusoidal vibrations were only applied to the HSV group, at a frequency of $30 \mathrm{~Hz}$ and an amplitude of $4 \mathrm{~mm}$. The external load used in HS and HSV was 5\% lower than that attained on the final day of familiarization, in order to en-

TABLE 2. Familiarization training and testing protocol.*

\begin{tabular}{|c|c|c|c|c|c|}
\hline Weeks & Period & Days & Sets & Repetitions & Recovery $\dagger$ \\
\hline 0 & Pre-Test & Thursday/Friday & \multicolumn{3}{|c|}{ Pre 10RM testing } \\
\hline \multirow[t]{3}{*}{1} & Familiarization training & Monday & 3 & 10 & 2 \\
\hline & & Wednesday & 3 & 10 & 2 \\
\hline & & Friday & 4 & 10 & 2 \\
\hline \multirow[t]{4}{*}{2} & Familiarization training & Monday & 4 & 10 & 2 \\
\hline & & Tuesday & 5 & 10 & 2 \\
\hline & & Thursday & 5 & 10 & 2 \\
\hline & & Friday & 5 & 10 & 2 \\
\hline \multirow[t]{3}{*}{3} & Post-Tests & Monday & & Post 10RM testing & \\
\hline & Tests* (HS and HSV) & Tuesday or Wednesday & 5 & 10 & 2 \\
\hline & & Thursday or Friday & 5 & 10 & 2 \\
\hline
\end{tabular}

\footnotetext{
* Two tests were performed in a randomized order with 48 hours of rest in between. $10 \mathrm{RM}=10$ repetitions maximum.
}

$\dagger$ Recovery between sets. 
TABLE 3. Food and energy composition of the pre-exercise snack.*

\begin{tabular}{|c|c|c|c|c|c|c|c|c|}
\hline \multirow[b]{2}{*}{ Ingredients } & \multirow[b]{2}{*}{ Amount (g) } & \multirow[b]{2}{*}{ Energy (kcal) } & \multicolumn{2}{|c|}{ Carbohydrate } & \multicolumn{2}{|c|}{ Protein } & \multicolumn{2}{|c|}{ Fat } \\
\hline & & & (g) & $\% \mathrm{TCV}$ & (g) & $\% \mathrm{TCV}$ & (g) & $\%$ TCV \\
\hline White bread & 80 & & & & & & & \\
\hline Cured ham & 20 & & & & & & & \\
\hline Orange juice & 250 & & & & & & & \\
\hline
\end{tabular}

$* \mathrm{TCV}=$ Total caloric value.

TABLE 4. Mean values ( $\pm S D$ ), mean difference, statistical significance, $95 \%$ confidence interval (CI), and effect size for the familiarization period.*

\begin{tabular}{cccccccc}
\hline & & & & \multicolumn{2}{c}{$95 \%$ CI } \\
\cline { 3 - 7 } Variable & Pre & Post & Diff Pre-Post & $p$ & Lower & Upper & Effect size \\
\hline $10 \mathrm{RM}$ & $51.52( \pm 10.7)$ & $60.2( \pm 11.3) \dagger$ & 8.71 & $<0.001$ & 7.43 & 9.97 & 3.504 \\
\hline
\end{tabular}

$* 10 \mathrm{RM}=10$ repetitions maximum.

$\dagger$ Significant difference with regard to the prevalue.

sure completion of the bout of 5 sets of 10 repetitions even during the application of WBV. Since the aim of this study was not to assess the effects of specific dietary manipulation, but rather the effects of exercise under normal conditions, subjects were allowed to eat a typical snack 1 hour before each test. The food composition and nutritional contribution of the snack are shown in Table 3.

\section{Energy Expenditure}

The pre-exercise $\dot{\mathrm{V}}_{2}$ (baseline resting metabolic rate) and RER for the HS and HSV groups were determined with the individuals standing up straight and relaxed for a 3minute period. During each type of exercise, including the rest intervals between sets and the first 4 minutes of recovery, $\mathrm{VO}_{2}$ and RER were measured continuously, recording data every 30 seconds. All oxygen consumption values were automatically converted by the software (Breeze Ex, MedGraphics, St. Paul, MN) into kcal per minute as an expression of EE at each stage. Subsequently, EE for the total bout of exercises (5 sets plus rest intervals) was calculated for HS and HSV (kcal per 11.5 minutes).

\section{Perceived Exertion and Heart Rate}

Heart rate was continuously monitored during training as well as at 5 minutes post exercise (Polar 510; Polar, Kempele, Finland). Following each set, subjects rate PE according to the Borg perceived exertion scale (CR10) (2), information on which was provided both verbally and in writing prior to training (18). Since subjects were wearing masks during training, and could not talk, they were asked at the end of each set to point at the appropriate $\mathrm{PE}$ rating on a copy of the scale placed on the wall throughout training. At 5 minutes post exercise, subjects rated their overall PE (15).

\section{Reproducibility of Measurements}

In a pilot study performed with 5 of the subjects in conditions similar to those of the study protocol, the interday reproducibility for variables $\mathrm{EE}$ and $\mathrm{RER}$ was found to be high. Intraclass correlation coefficients (ICC) for pre-exercise, exercise, and recovery conditions were as follows: EE: 0.96, 0.98, 0.95; and RER: 0.90, 0.91, 0.89.

\section{Statistical Analyses}

Traditional statistical methods were used to calculate mean values and $S D$. Sample normality was checked using the Shapiro-Wilk test. A Student's $t$-test was used to assess the effect of familiarization on the $10 \mathrm{RM}$ variable. The effect of independent variables (HS and HSV) on EE, RER, HR, and PE (dependent variables) was analyzed by analysis of variance (ANOVA). A Bonferroni correction was used to adjust $p$ values to reflect the number of contrasts performed. Effect size was calculated for paired variables (14). Rhea's scale was used to interpret the magnitude of effects in strength training: magnitude was thus classified as trivial $(<0.50)$, small $(0.50-1.25)$, moderate (1.25-1.9) or large ( $\geq 2)(30)$. Statistical significance was expressed as follows: $p \leq 0.05(*), p \leq 0.001$ (***). The significance level was set at $p \leq 0.05$ (*).The SPSS (version 11.5; SPSS, Inc., Chicago, IL) software package for Windows was used for all statistical tests.

\section{Results}

By the end of the familiarization period, subjects had experienced a significant $(p<0.001)$ increase in the 10RM load (16.8\%); values are shown in Table 4. However, in order to complete all 10-repetition sets for both HS and $\mathrm{HSV}$, the load had to be decreased somewhat, to 56.7 ( \pm 11.0) kg.

The values and statistical significance for the variables analyzed in this study for both conditions (HS and HSV) are described below; effect size and mean difference for all variables are shown in Table 5.

\section{Energy Expenditure}

Prior to exercise, there was no difference in EE between the HSV and HS groups $(1.63 \pm 0.30$ and $1.59 \pm 0.30$ $\mathrm{kcal} \cdot \mathrm{min}^{-1}$, respectively). Energy expenditure increased significantly during physical exercise and recovery in both groups $(p<0.001)$ (Figure 1$)$. There were no significant differences between pre-exercise RER values for the 2 groups ( $\mathrm{HSV}=0.96 \pm 0.10$ and $\mathrm{HS}=0.98 \pm 0.09$ ); RER increased significantly only in the HSV group during exercise $(p<0.001)$, and high levels were maintained during the recovery stage ( $p<0.001$ ) (Figure 2). Comparison of the HSV and HS groups during exercise 
TABLE 5. Effect size and mean difference values for energy expenditure (EE), respiratory exchange ratio (RER), heart rate (HR), and perceived exertion (PE).

\begin{tabular}{lrr}
\hline \multicolumn{1}{c}{ Variables } & Mean difference & Effect size \\
\hline EE pre-exercise & -0.04 & -0.100 \\
EE exercise & 0.66 & 1.565 \\
EE recovery & 0.60 & 1.184 \\
RER pre-exercise & -0.02 & -0.243 \\
RER exercise & 0.07 & 1.695 \\
RER recovery & 0.01 & 1.088 \\
HR, first set & 0.10 & 0.007 \\
HR, second set & 1.30 & 0.095 \\
HR, third set & 6.20 & 0.516 \\
HR, fourth set & 1.90 & 0.126 \\
HR, fifth set & 3.10 & 0.179 \\
HR recovery & -3.90 & -0.220 \\
PE, first set & 0.60 & 0.643 \\
PE, second set & 1.00 & 0.805 \\
PE, third set & 0.40 & 0.404 \\
PE, fourth set & 0.90 & 0.690 \\
PE, fifth set & 0.80 & 0.873 \\
PE recovery & 0.60 & 0.586 \\
\hline
\end{tabular}

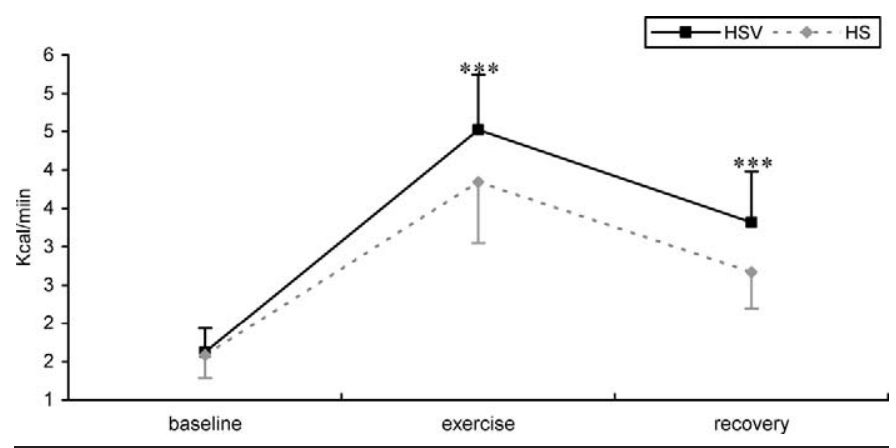

FIGURE 1. Comparison of energy expenditure for half-squat with vibration (HSV) and half squat (HS) at 3 different time periods. *** Indicates significant difference between HSV and HS groups $(p<0.001)$.

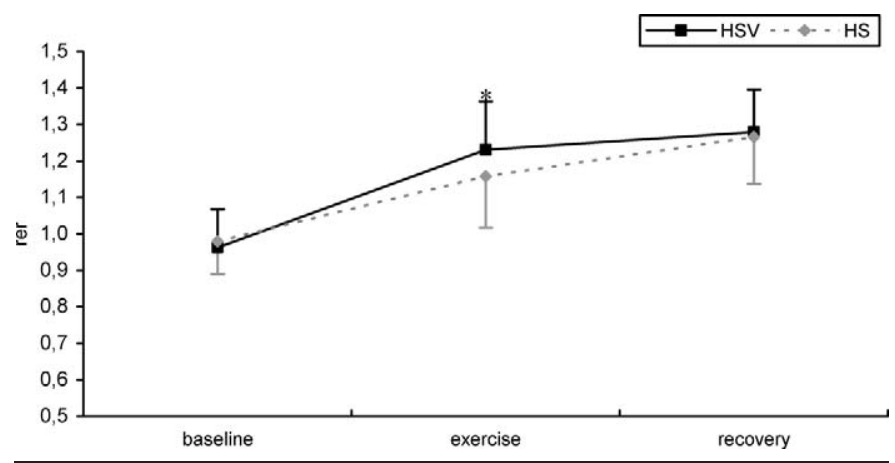

FIGURE 2. Comparison of respiratory exchange ratio for halfsquat with vibration (HSV) and half squat (HS) at 3 different time periods. * Indicates significant difference between HSV and HS groups $(p<0.05)$.

showed that the application of WBV had a significant effect on $\operatorname{EE~}\left(4.50 \pm 0.72\right.$ vs. $3.84 \pm 0.79 \mathrm{kcal} \cdot \mathrm{min}^{-1}, p<$ $0.001)$ and $\operatorname{RER}(1.23 \pm 0.13$ vs. $1.16 \pm 0.10, p<0.05)$. Similarly, during the recovery stage, the HSV group also had displayed higher EE than the HS group (3.30 \pm 0.66 vs. $2.70 \pm 0.47 \mathrm{kcal} \cdot \mathrm{min}^{-1}$ respectively, $p<0.001$ ), whereas the RER for the 2 groups evened out during the recovery period $(\mathrm{HSV}=1.28 \pm 0.11$ and $\mathrm{HS}=1.27 \pm 0.13$ ).

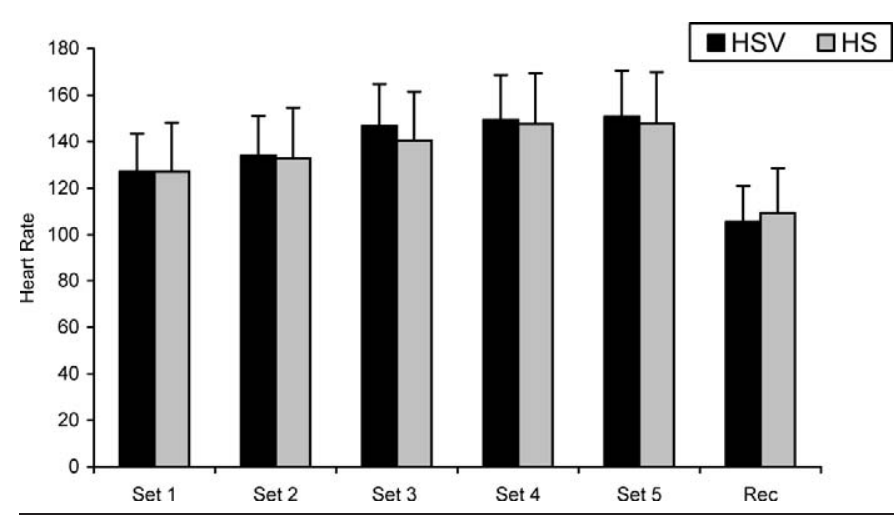

FigURE 3. Heart rate mean values in response to half-squat with vibration (HSV) and half squat (HS) during each training set and at 5 minutes into recovery.

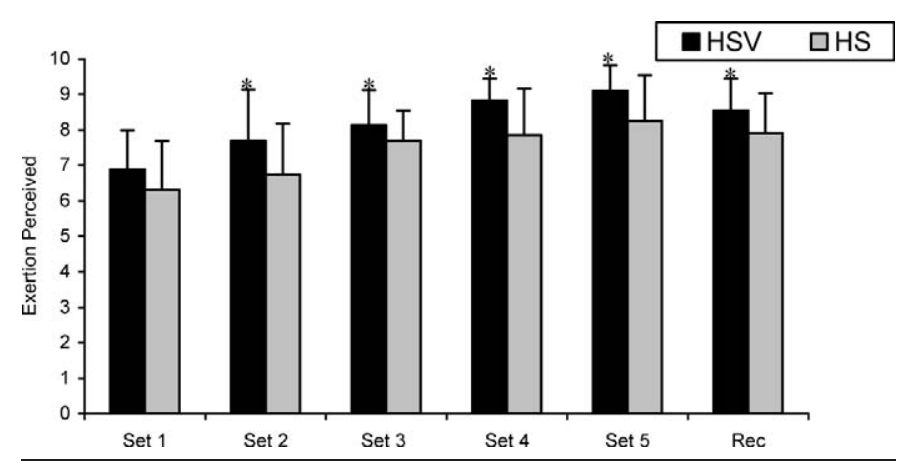

Figure 4. Perceived exertion mean values in response to half-squat with vibration (HSV) and half squat (HS) during each training set, and global perceived exertion at 5 minutes into recovery. * Indicates significant difference between HSV and HS groups $(p<0.05)$.

Total EE for the HSV bout was significantly higher than for the HS bout $(52.0 \pm 8.34$ vs. $44.2 \pm 9.10 \mathrm{kcal}, p<$ $0.05)$.

\section{Heart Rate}

Heart rate values were higher for the HSV group during each of the 5 training sets, and slightly lower 5 minutes into the recovery stage; however, differences between groups were not significant in either case (Figure 3).

\section{Perceived Exertion}

The PE of the HSV group was significantly higher from the second training set $(p<0.05)$ (Figure 4$)$. The overall perception of HSV training, recorded after 5 minutes of passive recovery, was also higher than for HS training ( $p$ $<0.05$ ) (Figure 4).

\section{Discussion}

This study assessed the effect of whole-body vibration, at a predetermined amplitude and frequency, on energy expenditure when performing half-squat exercises with an external load of 10RM. Assessment was based on continuous indirect calorimetry throughout the pre-exercise rest period, exercise, rest intervals between sets, and the acute recovery stage. As expected, EE during HSV was significantly higher than during HS alone; HSV was found to provide a stimulus similar to that experienced by a subject of similar weight during moderate walking at $4 \mathrm{~km}$ per hour (28). Subjects' perceived exertion rating was also higher for HSV. 
Although familiarization training was intended simply to familiarize subjects with the specific movements required for HS exercises, there was a significant change in $10 \mathrm{RM}$ as a result of familiarization. This is readily attributable to the fact that most subjects did not previously perform HS as part of their routine training; it is widely documented that changes in the type of exercise, number of sets, or repetitions can prompt a short-term increase in strength (29).

Energy expenditure during HSV training was $17 \%$ higher than for HS. This appears to confirm that the high metabolic load resulting from resistance training (10RM) (25) may be complemented by additional neuroendocrine stimulation derived from the involuntary contractions provoked by the vibrations (32).

Although Rittweger et al. (32) obtained energy-expenditure equivalents for resistance exercise with WBV, they studied exercise (squatting with or without load) with added vibration only over 1 uninterrupted 3-minute period. This type of exercise cannot readily be repeated or combined with other regular resistance-training exercises. The present study recorded significant energy-expenditure increases as a result of applying vibration, although increases were less marked than those reported by Rittweger et al. (32). In general terms, results are comparable, and the real exercise-sets studied here could be viably included in a resistance training protocol, since they cover the active phase, the rest intervals between sets, a commonly-used time period, external load, and tolerable vibrations.

The 6\% increase in RER observed in HSV compared to HS might reflect the greater intensity of work resulting from WBV, and the greater need to disperse $\mathrm{CO}_{2}$ and other metabolic products. Even though increased use of carbohydrates has been reported in predominant anaerobic glycogenolytic pathways during this type of strength training (36), there is also high lactate production (27). Acidosis would not make RER a viable predictor of substrate oxidation, since its buffering, via bicarbonate, increases the excretion of $\mathrm{CO}_{2}$ without increasing $\mathrm{O}_{2}$ uptake, thus raising RER (28).

With regard to the recovery period, 1 limitation of the present study was the short duration of the period analyzed (4 minutes) and the lack of lactic acid monitoring, which meant that the behavior of EE and RER after the acute recovery period could not be investigated.

During the 4-minute recovery stage, energy expenditure was $22 \%$ higher in the HSV group than in the HS group, reflecting additional post-exercise metabolic activity following the application of WBV. It has been suggested that hypertrophy strength training generates sustained increases in anabolizing hormone levels, and that the magnitude of this effect is correlated with the lactosemia achieved during effort (21), reflecting the neuroendocrine response necessary for repair processes (12). Moreover, the existence of a correlation between the intensity of resistance training and the magnitude and duration of higher post-exercise oxygen consumption levels $(3,37)$ suggests that application of WBV may optimize these effects and therefore optimize post-exercise energy expenditure.

The RER values in both exercise groups (HSV and HS) were higher (28 and $27 \%$, respectively) during the recovery stage than pre-exercise, with no significant difference between the 2 groups. As indicated earlier, the post-exercise lactic acid load means that these RER values could not be used as indicators of the use of specific substrates.
However, it has been reported that a high RER is observed in the first few minutes of recovery following resistance exercises; from then on it decreases significantly to below baseline values (1) and even below the RER produced in the recovery stage following treadmill exercises (7). This may point to increased lipid oxidation after resistance exercises, when lactic acid values fall without modifying the RER (1). In this respect, it has been suggested that intense exercise, a decisive factor in glucogenic depletion, is likely to induce a compensatory carbohydrate storing mechanism while also increasing lipid oxidation during recovery (8). If this is so, the application of vibrations to hypertrophy strength training could be considered to add more intensity to the effort, thus perhaps increasing post-exercise fat oxidation.

The higher heart rate recorded in the HSV group, although not statistically significant, agrees with the findings of other studies, which report increased HR during a short period of exposure to WBV $(22,35)$. After 5 minutes of recovery time, the heart rate of the HSV group was lower than that of the HS group, suggesting a faster recovery. Guignard, similarly, notes increased HR during exposure to WBV, followed by a rapid return to HR baseline values in the acute recovery stage (20).

$\mathrm{PE}$ was significantly higher during HSV following the first set, presenting high values in the last 2 sets, reflecting greater intensity in this exercise. A strong correlation has been reported between the levels of lactate and PE in resistance training with short recovery times (26). Although lactic acid levels were not measured here, it has been suggested that the application of vibration reduces levels of muscular oxygenation in comparison to work carried out without vibration (42), which might be associated with increased activation of the lactic anaerobic pathway.

In conclusion, this study presents evidence that a bout of resistance training consisting of $\mathrm{HS}$ with application of WBV may produce a higher metabolic load, associated with a higher PE, than the same training without WBV. The result would be an increase in EE during and after effort, and possibly an increase in the post-effort use of substrates.

\section{Practical Applications}

This study indicates that subjects performing conventional HS training optimized energy expenditure through the application of WBV, achieving greater metabolic stimulation and greater energy expenditure. This form of exercise would not only be advantageous from an energyexpenditure standpoint, but could also be readily included as part of a resistance training program. This would require careful preplanning of external loads, and the collaboration of a multidisciplinary team. Where the aim is to lose body fat mass, WBV programs could be combined with other physical exercise and with dietary changes, to favor the achievement of a negative energy balance and to maintain or increase muscle mass.

\section{REFERENCES}

1. Binzen, C.A., P.D. Swan, And M.M. Manore. Postexercise oxygen consumption and substrate use after resistance exercise in women. Med. Sci. Sports Exerc. 33:932-938. 2001.

2. Borg, G. The Borg CR10 Scale: Borgs Perceived Exertion and Pain Scales. Champaign, IL: Human Kinetics, 1998.

3. BorsheIm, E., AND R. BAHR. Effect of exercise intensity, duration and mode on post-exercise oxygen consumption. Sports Med. 33:1037-1060. 2003.

4. Bosco, C., M. Cardinale, O. Tsarpela, R. Colli, J. Tihanyi, S.P. von DUVILLARD, AND A. VIRU. The influence of whole body vibration on jumping performance. Biol. Sport 15:157-164. 1998. 
5. Bosco, C., R. Colli, E. Introini, M. Cardinale, M. Iacovelli, J. TihanyI, S.P. Von Duvillard, AND A. Viru. Adaptive responses of human skeletal muscle to vibration exposure. Clin. Physiol. 16:317-322. 1999.

6. Bosco, C., M. Iacovelli, O. Tsarpela, M. Cardinale, M. Bonifazi, J. Tihanyi, M. Viru, A. De Lorenzo, and A. ViRu. Hormonal responses to whole-body vibration in men. Eur. J. Appl. Physiol. 81:449-454. 2000.

7. Braun, W.A., W.E. Hawthorne, and M.M. Markofski. Acute EPOC response in women to circuit training and treadmill exercise of matched oxygen consumption. Eur. J. Appl. Physiol. 94:500-504. 2005.

8. BROOKS, G.A., AND J. MERCIER. Balance of carbohydrate and lipid utilization during exercise: The crossover concept. J. Appl. Physiol. 76:22532261. 1994

9. Brozek, J., F. Grande, J.T. Anderson, and A. Keys. Densitometric analysis of body composition: Revision of some quantitative assumptions. Ann. N.Y. Acad. Sci. 110:113-140. 1963.

10. Bryner, R.W., I.H. Ulrich, J. SAUers, D. Donley, G. Hornsby, M. KoLAR, AND R. YEATER. Effects of resistance vs. aerobic training combined with an 800 calorie liquid diet on lean body mass and resting metabolic rate. J. Am. Coll. Nutr. 18:115-121. 1999.

11. CAMPBell, W.W., M.C. CRim, V.R. YounG, AND W.J. Evans. Increased energy requirements and changes in body composition with resistance training in older adults. Am. J. Clin. Nutr. 60:167-175. 1994

12. Chesley, A., J.D. MacDougall, M.A. Tarnopolsky, S.A. Atkinson, AND K. SMith. Changes in human muscle protein synthesis after resistance exercise. J. Appl. Physiol. 73:1383-1388. 1992.

13. Cochrane, D.J., S.J. LEGG, AND M.J. Hooker. The short-term effect of whole body vibration training on vertical jump, sprint, and agility performance. J. Strength Cond. Res. 18:828-832. 2004.

14. Cohen, J. Statistical Power Analysis for the Behavioral Sciences (2nd ed.). Hillsdale, NJ: L. Erlbaum Associates, 1998. pp. xxi, 567.

15. Day, M.L., M.R. McGuigan, G. Brice, and C. Foster. Monitoring exercise intensity during resistance training using the session RPE scale. J. Strength Cond. Res. 18:353-358. 2004.

16. Delecluse, C., M. Roelants, and S. Verschueren. Strength increase after Whole Body Vibration compared with resistance training. Med. Sci. Sports Exerc. 35:1033-1041. 2003.

17. Durnin, J., AND J. Womersley. Body fat assessed from total body density and its estimation from skinfold thickness: Measurements on 481 men and women aged from 16 to 72 years. Br. J. Nutr. 32:77-97. 1974.

18. Gearhart, R.E., F.L. Goss, K.M. LAGally, J.M. JaKicic, J. Gallagher, AND R.J. RoBeRTSON. Standardized scaling procedures for rating perceived exertion during resistance exercise. J. Strength Cond. Res. 15:320325. 2001.

19. Gonzalez-Badillo, J.J. Modelos de planificación y organización del entrenamiento de fuerza. In: $V$ Congreso Internacional sobre entrenamiento de Fuerza: el entrenamiento de fuerza como clave del rendimiento deportivo; León, Spain, 1, 2 y 3 de Octubre, 1998.

20. GuignaRd, J.C. A note on the heart rate during low-frequency wholebody vibration. Scientific Memorandum No. 58. Royal Air Force Institute of Aviation Medicine, Farnborough. 1964.

21. HÄKKINEN, K., AND A. PAKARINen. Acute hormonal responses to two different fatiguing heavy-resistance protocols in male athletes. J. Appl. Physiol. 74:882-887. 1993

22. HoRnick, R.J., AND N.M. Lefritz. A study and review of human response to prolonged random vibration. Hum. Factors 8:481-492. 1966.

23. Issurin, V.B., D.G. LiebermanN, AND G. TENENBAum. Effect of vibratory stimulation training on maximal force and flexibility. J. Sports Sci. 12: 561-566. 1994.
24. Kraemer, W.J., AND A.C. Fry. Strength testing: Development and evaluation methodology. In: Physiological Assessment of Human Fitness. P. Maud and C. Foster, eds. Champaign, IL: Human Kinetics. 1995.

25. Kraemer, W.J., L. Marchiteldi, S.E. Gordon, E. Harman, J.E. DziaDos, R. Mello, P. Frykman, D. McCurRy, and S. Fleck. Hormonal and growth factor responses to heavy resistance exercise protocols. J. Appl. Physiol. 69:1442-1450. 1990.

26. Kraemer, W.J., B.J. Noble, M.J. Clark, and B.W. Culver. Physiologic responses to heavy resistance exercise with very short rest periods. Int. J. Sports Med. 8:247-252. 1987.

27. MacDougall, J.D., A.L. Hicks, J.R. MacDonald, R.S. McKelvie, H.J. GREEN, AND K.M. SMITH. Muscle performance and enzymatic adaptations to sprint interval training. J. Appl. Physiol. 84:2138-2142. 1998.

28. McARdLE, W.D., F.I. Katch, AND V.L. Katch. Exercise Physiology Energy, Nutrition, and Human Performance (5th ed.). Philadelphia: Lippincott, Williams and Wilkins. 2001.

29. Poliquin, C. Five steps to increasing the effectiveness of your strength training program. Natl. Strength Cond Assoc. J. 10:34-39. 1988.

30. RHEA, M.R. Determining the magnitude of treatment effects in strength training research through the use of the effect size. J. Strength Cond. Res. 18:918-920. 2004.

31. Rittweger, J., K. Just, K. Kautzsch, P. Reeg, and D. Felsenberg. Treatment of chronic lower back pain with lumbar extension and wholebody vibration exercise: A randomized controlled trial. Spine 27:18291834. 2002.

32. Rittweger, J., H. Schiessl, ANd D. Felsenderg. Oxygen uptake during whole-body vibration exercise: Comparison with squatting as a slow voluntary movement. Eur. J. Appl. Physiol. 86:169-173. 2001.

33. RonNestaD, B.R. Comparing the performance-enhancing effects of squats on a vibration platform with conventional squats in recreationally resistance-trained men. J. Strength Cond. Res. 18:839-845. 2004.

34. Rubin, C., R. Recker, D. Cullen, J. Ryaby, J. McCabe, and K. McLEOD. Prevention of postmenopausal bone loss by a low-magnitude, highfrequency mechanical stimuli: A clinical trial assessing compliance, efficacy, and safety. J. Bone Miner. Res. 19:343-351. 2004.

35. RYLANDS, J.M. The effect of a long duration exposure to noise and vibration on task performance. Master's thesis, University of London, 1985.

36. Tesch, P.A., E.B. Colliander, And P. Kaiser. Muscle metabolism during intense, heavy-resistance exercise. Eur. J. Appl. Physiol. Occup. Physiol. 55:362-366. 1986.

37. Thornton, M.K., AND J.A. Potteiger. Effects of resistance exercise bouts of different intensities but equal work on EPOC. Med. Sci. Sports Exerc. 34:715-722. 2002

38. Verschueren, S., M. Roelants, C. Delecluse, S. Swinnen, D. VanDERSCHUEREN, AND S. BOONEN. Effect of 6-month whole body vibration training on hip density, muscle strength, and postural control in postmenopausal women: A randomized controlled pilot study. J. Bone Miner. Res. 19:352-359. 2004.

39. WHO/FAO. 2001 Report of a Joint FAO/WHO/UNU Expert Consultation in Human Energy Requirements. FAO Food and Nutrition Technical Paper Series, No. 1. Roma: 2001. p. 4.

40. WHO/FAO 2003 Joint WHO/FAO Expert Consultation in Diet, Nutrition and the Prevention of Chronic Diseases. WHO Technical Report Series 916. Geneva: 2003. p. 64.

41. Winett, R.A., AND R.N. CARPinelli. Potential health-related benefits of resistance training. Prev. Med. 33:503-513. 2001.

42. Yamada, E., T. Kusaka, K. Miyamoto, S. Tanaka, S. Morita, S. TaNAKA, S. TsujI, S. Mori, H. Norimatsu, AND S. ITOH. Vastus lateralis oxygenation and blood volume measured by near-infrared spectroscopy during whole body vibration. Clin. Physiol. Funct. Imaging 25:203-208. 2005.

Address correspondence to Marzo Edir Da Silva, PhD, pit_researcher@yahoo.es. 